Canadian Art Review

\title{
Le détournement du commissariat : l'exposition chorégraphique chez Boris Charmatz et Xavier Le Roy
}

\section{Véronique Hudon}

Volume 43, numéro 2, 2018

What is Critical Curating?

Qu'est-ce que le commissariat engagé ?

URI : https://id.erudit.org/iderudit/1054383ar

DOI : https://doi.org/10.7202/1054383ar

Aller au sommaire du numéro

\section{Éditeur(s)}

UAAC-AAUC (University Art Association of Canada | Association d'art des universités du Canada)

ISSN

0315-9906 (imprimé)

1918-4778 (numérique)

Découvrir la revue

Citer cet article

Hudon, V. (2018). Le détournement du commissariat : l'exposition chorégraphique chez Boris Charmatz et Xavier Le Roy. RACAR : Revue d'art canadienne / Canadian Art Review, 43(2), 57-72.

https://doi.org/10.7202/1054383ar

\section{Résumé de l'article}

Today, it is not unusual to see performing artists present exhibitions in galleries or museums. The French choreographers Boris Charmatz and Xavier Le Roy each revisit the format of the exhibition as a performative site, rather than a space devoted to artistic objects. At the border between curating and artistic practice, their work questions the museum space as a place for the production of knowledge. Through living practice, choreographers redefine the work of art's modes of production, mediation, and reception, while also reformulating aesthetic experience through the coexistence of bodies, documents, and artistic media in the museum space. By reflecting on the critical potential of Boris Charmatz's Flip Book exhibitions and Xavier Le Roy's "Retrospective," this article seeks to define their curatorial strategies, as well as the political consequences of their engagement in the institution.
Tous droits réservés (C) UAAC-AAUC (University Art Association of Canada | Association d'art des universités du Canada), 2018
Ce document est protégé par la loi sur le droit d'auteur. L'utilisation des services d'Érudit (y compris la reproduction) est assujettie à sa politique d'utilisation que vous pouvez consulter en ligne.

https://apropos.erudit.org/fr/usagers/politique-dutilisation/ 


\title{
Le détournement du commissariat: l'exposition chorégraphique chez Boris Charmatz et Xavier Le Roy
}

\author{
Véronique Hudon
}

Today, it is not unusual to see performing artists present exhibitions in galleries or museums. The French choreographers Boris Charmatz and Xavier Le Roy each revisit the format of the exhibition as a performative site, rather than a space devoted to artistic objects. At the border between curating and artistic practice, their work questions the museum space as a place for the production of knowledge. Through living practice, choreographers redefine the work of art's modes of production, mediation, and reception, while also reformulating aesthetic experience through the coexistence of bodies, documents, and artistic media in the museum space. By reflecting on the critical potential of Boris Charmatz's Flip Book exhibitions and Xavier Le Roy's "Retrospective," this article seeks to define their curatorial strategies, as well as the political consequences of their engagement in the institution.

Véronique Hudon est commissaire, critique d'art et doctorante en Études et pratiques des arts à I'Université du Québec à Montréal. -veronique.hudon@gmail.com

1. En 2014, la revue Dance Research publie un numéro spécial intitulé "Dance in the Museum», sous la direction de Mark Franko et d'André Lepecki. Dans leur texte d'introduction, «Editor's Note: Dance in the Museum», les auteurs observent que l'intérêt actuel des musées pour la danse est fort différent des expérimentations des années 1960 qui ont eu lieu notamment à New York: «the scale, pre-
La danse contemporaine est à nouveau présente ${ }^{1}$ dans les musées et les galeries d'art avec des ambitions toutefois différentes, puisque la danse et les arts vivants sont en voie de s'implanter de manière durable dans les musées par un commissariat permanent qui inclut la programmation et la gestion d'espaces spécifiques attribués à l'art vivant. En continuité, certains chorégraphes revisiteraient le format de l'exposition, en remettant en question ses cadres perceptuels traditionnels et en renouvelant les relations entre l'artiste, le public et l'œuvre. En repensant l'exposition à partir de la pratique du danseur, Boris Charmatz et Xavier Le Roy se situent à cette frontière du commissariat et de la pratique artistique. À travers leurs «œuvres incarnées», c'est-àdire formées de corps vivants en action, ces deux chorégraphes interrogent le bagage historique, symbolique et commissarial du musée. Le travail qu'accomplit Boris Charmatz depuis qu'il a redéfini l'identité du Centre national chorégraphique de Rennes et de Bretagne, en 2009, en le transformant en Musée de la danse est exemplaire. Dans le manifeste qui appuie cette nouvelle identité, Charmatz s'engage à renouveler la muséologie à partir de la pratique vivante et, plus spécialement, de la danse. La méthodologie muséale qu'il préconise vise à faire émerger le savoir non pas de l'analyse de l'objet a posteriori, mais de la pratique artistique elle-même: la connaissance découle de l'expérimentation. Charmatz et le Musée de la danse proposent depuis les dernières années plusieurs «expositions dansées» en collaboration avec d'autres musées et centres d'art. Notons la collaboration avec le Tate Modern lors de l'événement IfTate Modern was Musée de la danse? en 2015 | fig. 1 | et l'événement Three Collective Gestures, présenté au MoMA en 2013, dont il sera ici question. Dans le cadre de ces divers événements, le Musée de la danse présente plusieurs projets au sein du musée. Ainsi, l'événement Three Collective Gestures regroupe trois projets: 20 danseurs pour le xx siècle (2012), Flip Book (2009) et Levée des conflits extended (2010). ${ }^{2}$ Dans cet article, je m'attarderai plus spécifiquement sur la pièce Flip Book (2009), ${ }^{3}$ qui a aussi fait l'objet d'une adaptation au Tate Modern lors de l'événement The Tanks: Art in Action en 2012. | fig. 2 | Flip Book par le biais de la figure de Merce Cunningham, présente un réinvestissement humain de l'archive et de l'histoire de la danse.

Le chorégraphe Xavier Le Roy, quant à lui, présente en 2012 à La Fondació Antoni Tàpies de Barcelone une exposition intitulée "Rétrospective» 4 fig. $3 \mid$ qui, selon la théoricienne Bojana Cvejić, «détourne le format de rétrospective aussi bien du point de vue de l'histoire de l'art que des pratiques 
valence, and consistency in presenting dance currently... suggest that something else is taking place right now». Claire Bishop, qui participe au numéro, exprime aussi la particularité de la situation, dans son article «The perils and possibilities of dance in the museum», en affirmant que les exemples précédents dans l'histoire du musée et des arts vivants sont «completely different in tenor and ambition to what is going on today». Voir Mark Franko et André Lepecki, dir., Dance Research Journal, vol. $46, n^{\circ} 3,2014$, p. 3-4.

2. Levée des conflits est une pièce créée en 2010, dans laquelle vingtquatre danseurs se jettent, l'un après l'autre, dans un «canon chorégraphique» jusqu'à l'épuisement. Ils se débattent et s'ébattent dans une dépense d'intensité. Dédiée à Roland Barthes et à son concept de «neutre», la pièce cherche, comme son titre l'indique, à «lever des conflits» dans un cycle qui renvoie sans cesse de l'individuel au collectif.

3. Notez que pour l'ensemble de l'article les photographies sont tirées de présentations et d'adaptations du Tate Modern: Roman photo lors de l'événement IfTate Modern was Musée de la danse? (2015) et Flip Book lors de l'événement The Tanks: Art in Action (2012), qui soulignait l'ouverture de l'espace des Tanks consacré aux arts vivants, à la performance, aux installations et aux vidéos d'art actuel. Les photographies donnent à comprendre les pièces et les procédés qui demeurent les mêmes.

4. Aujourd'hui, l'exposition a été présentée maintes fois dans plusieurs musées: Musée de la danse, Centre Pompidou, Museu de Arte do Rio, MoMA PS1, entre autres.

5. Bojana Cvejić, dir., «Rétrospective» par Xavier Le Roy, Dijon, Les Presses du réel, 2014, p. 10.

6. Cité dans ibid.

7. Terme qu'emploie notamment Jacques Rancière dans son livre Lespectateur émancipé (2000), Paris, La Fabrique, 2008.

8. Cette désignation est souvent contestée par les artistes, les critiques et les théoriciens. À ce sujet, il faut lire l'article de Jeroen Fabius qui retrace les différentes polémiques et discussions autour d'une telle nomination. Jeroen Fabius, «The missing history of (not)conceptual dance», dans Merel Heering, Ruth Naber, Bianca Nieuwboer et Liesbeth Wildschut, dir., Danswetenschap in Nederland, vol. 7, Amsterdam, Vereniging voor Dansonderzoek, 2012.

9. Ibid. curatoriales». ${ }^{5}$ «Rétrospective» revisite le format de l'exposition en offrant aux danseurs de s'approprier les pièces solos de Le Roy, tout en les soumettant à un protocole spécifique d'actions qui rythme l'exposition. La rétrospective est loin d'offrir un parcours linéaire et unifié de la démarche artistique de Le Roy. Elle met plutôt de l'avant une forme de partage collectif qui tend à effacer la figure de l'artiste au lieu de la glorifier. La mémoire des danseurs se substitue ainsi au discours historique, alors que des documents d'archives et des artefacts deviennent des outils pour évoquer les œuvres de Le Roy: la «situation [est] mise en scène au moyen d'artifices» ${ }^{6}$ afin de convoquer la mémoire collective et individuelle.

Ces deux chorégraphes vont me permettre de montrer comment la danse peut faire de l'exposition un dispositif performatif, car ils substituent aux objets d'art, que l'on retrouve traditionnellement dans les musées, des corps en mouvement et en action. Ils ne rejettent pas pour autant l'objet. Ils cherchent davantage à réfléchir sur la coexistence des corps, des documents d'archives et des médiums dans l'espace muséal. À ce titre, les œuvres de Charmatz et Le Roy que j'analyserai ici prennent en charge les modes de production, tout autant que les modes de médiation, de présentation et de diffusion. On verra que le corps du danseur joue sur l'ambiguïté de son propre statut, entre sujet et objet, et que le spectateur est appelé à devenir un «interprète actif $^{7}$ dont la participation est essentielle. L'exposition devient ainsi un site où le chorégraphe, le danseur, ainsi que le spectateur génèrent une expérience renouvelée du musée. La pratique de Charmatz et Le Roy serait ainsi motivée par un parti pris critique qui s'inscrirait dans une perspective commissariale engagée.

Leur engagement fait de la pratique chorégraphique un outil pour lire autrement les histoires des arts. L'analyse du dispositif performatif de Flip Book de Charmatz, qui met à nu les processus de création de l'œuvre même, permettra de comprendre l'importance de la transmission orale et corporelle en danse. Cette approche ouvre sur une histoire autre dans laquelle la mémoire et l'oralité jouent un rôle central. L'exposition «Rétrospective» de Le Roy permet d'analyser l'engagement des danseurs à même de raconter leurs récits professionnel et personnel. Ces deux artistes rendent visibles et explicites les processus menant à l'œuvre dansée, et renouent ainsi avec les fonctions sociales et politiques de la pratique artistique. Ils proposent une expérience esthétique au-delà de l'objet qui, dans un contexte muséal, tend à être déconnectée des processus normaux de la vie. Ils détournent également les méthodologies muséales plus traditionnelles en leur substituant une approche performative en continuité avec l'expérience vécue.

\section{De la danse conceptuelleà l'exposition chorégraphique}

Le travail de Boris Charmatz et de Xavier Le Roy est souvent désigné comme de la «danse conceptuelle», ${ }^{8}$ une pratique chorégraphique qui émerge dans le milieu des années 1990 et dont certains principes rappellent l'art conceptuel des années 1960 et 1970. Dans son article, «The missing history of (not) conceptual dance » (2012), ${ }^{9}$ le chercheur Jeroen Fabius analyse l'émergence du terme «danse conceptuelle» et les débats auxquels a donné lieu cette désignation. Il considère le critique autrichien Helmut Ploebst comme le 
Figure 1. Boris Charmatz et le Musée de la danse, Roman Photo tiré de Flip Book, 2015, adapté par Olivia Grandville pour un groupe de non danseurs londoniens, Londres, Tate Modern. Photo Brotherton Lock, ( Tate.

Figure 2. Boris Charmatz et le Musée de la danse, Flip Book, 25-29 Septembre 2012, en performance lors de l'événement The Tanks: Art in Action, Londres, Tate Modern. Photo: Tate, (c) Tate.

Figure 3. Xavier Le Roy, "Rétrospective », New York, MoMA PS1. Photo: Matthew Spetimus,

(c) Courtoisie de l'artiste et du MoMA PS1.
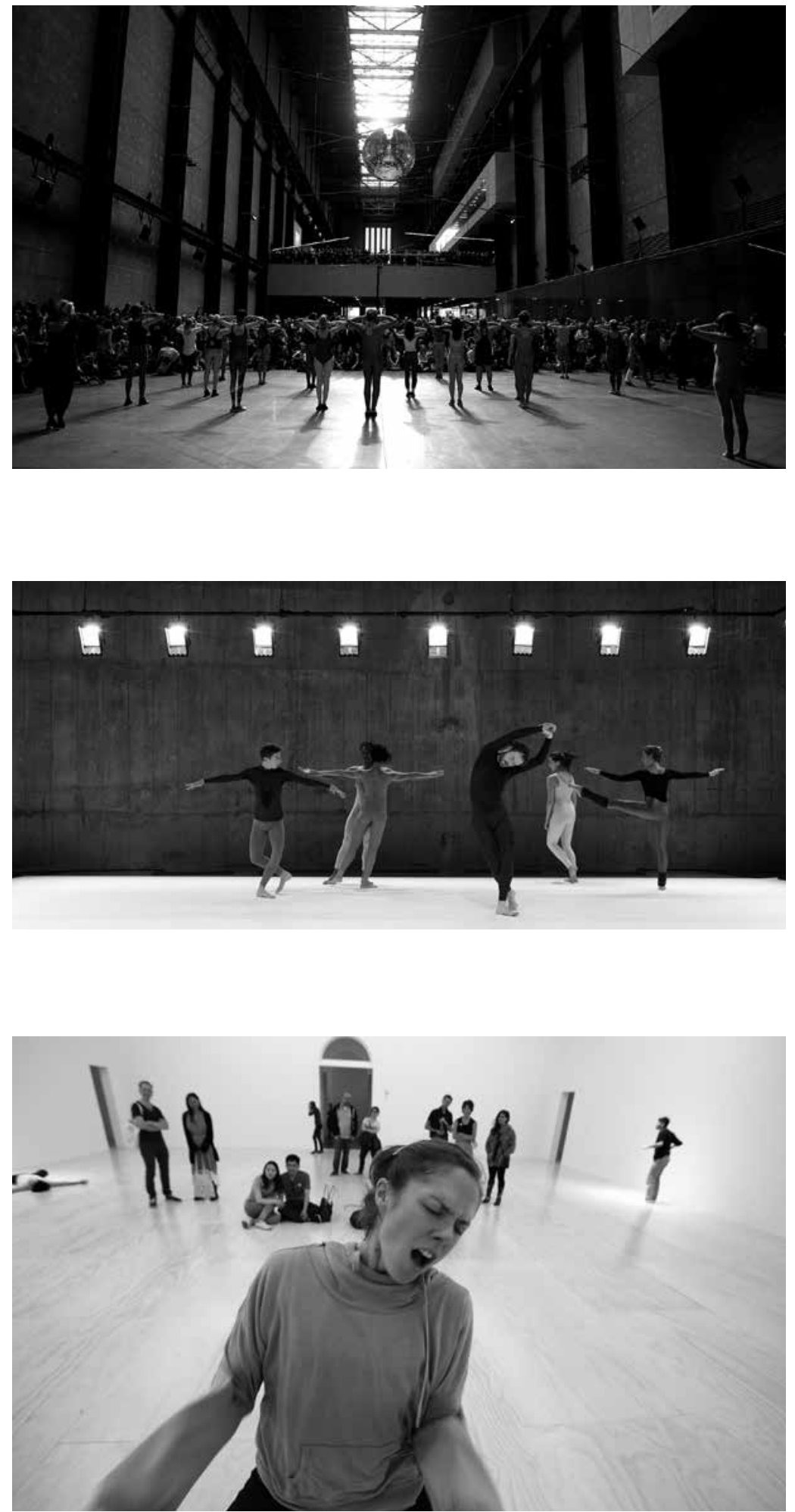
10. Helmut Ploebst, No Wind No Word. New choreography in the society of the spectacle: 9 portraits, Munich, K. Kieser, 2001.

11. L'opposition à cette nomination chez les chorégraphes ou les théoriciens vient du fait qu'elle est perçue péjorativement, en ce qu'elle invaliderait le fait qu'elle est de la danse: la nomination «non-danse», donnée par certains critiques peu réceptifs à cet art, a certainement contribué à cette position défensive. Voir Johannes Birringer, "Dance and Not Dance», Performing Arts Journal, vol. 80, mai 2005, p. 10-27.

12. Claire Bishop, "Je ne veux pas de rétrospective! Xavier Le Roy et l'exposition en tant que médium», dans Cvejić, dir., op. cit. p. 107.

13. Mathieu Copeland, «Chorégraphier l'exposition: une exposition se réalisant en tout lieu, en tout temps, avec et pour tous», dans Mathieu Copeland, dir., Chorégraphier l'exposition, Dijon, Les Presses du réel, 2013. premier à recenser le travail d'artistes en danse conceptuelle, dans son livre No Wind No Word (2001), dédié au travail d'Emio Greco/PC, de João Fiadeiro, de Vera Mantero, de Boris Charmatz, de Jérôme Bel, de Xavier Le Roy, de Raimund Hoghe, de Maria La Ribot et de Benoît Lachambre. ${ }^{10}$ Bien que l'expression "danse conceptuelle» ne fasse pas l'unanimité, comme le souligne Jeroen Fabius au fil de ces recensions, elle persiste dans le temps et c'est pourquoi il apparaît légitime d'explorer les relations esthétiques entre la danse et l'art conceptuel. ${ }^{11}$ En effet, les chorégraphes partagent avec les artistes conceptuels une approche centrée sur l'idée, la perception, l'interaction avec les spectateurs et se tournent vers des pratiques expérimentales et hybrides, tout en exerçant une critique des institutions. La pièce chorégraphique est abordée comme concept et le rôle du chorégraphe en tant qu'auteur est remis en question. L'art conceptuel a aussi exercé, dans le domaine des arts visuels, une forte critique de l'institution muséale, de ses dispositifs de présentation et de ses méthodes de pérennisation, ce qui a poussé certains artistes à revisiter l'exposition et à se l'approprier comme si c'était un médium artistique. C'est le cas notamment de «Working Drawings and Other Visible Things on Paper Not Necessarily Meant to be Viewed as Art», présentée en 1966 à la School of Visual Arts de New York. Réunissant plusieurs artistes phares de l'art conceptuel sous l'initiative de Mel Bochner, cette exposition présente les ébauches et les carnets de notes de l'exposition elle-même et met ainsi à nu ses processus de création. Ainsi, l'exposition comme médium artistique est utilisée afin de créer des projets expérimentaux où les modes de production se substituent aux objets artistiques. À la suite des artistes conceptuels, les chorégraphes s'emparent eux aussi de l'exposition en tant que médium à revisiter. L'historienne de l'art Claire Bishop souligne que certains chorégraphes, notamment Xavier Le Roy, «traitent [...] l'exposition comme une forme en soi, comme un médium qui n'est pas nécessairement une fin ou un aboutissement, mais le point de départ d'un processus de réinvention perpétuelle». ${ }^{12}$ En effet, les pièces chorégraphiques sont en mouvement dans l'espace et le temps. Ainsi, le cadre de l'exposition est constamment renouvelé par l'œuvre dansée.

Les chorégraphes rejouent aussi autrement certains principes au cœur de l'art conceptuel, mais dont il faut d'emblée soulever certains paradoxes. The Anxious Object d'Harold Rosenberg (1966), Six Years: The Dematerialization of the Art Object de Lucy Lippard (1973), ou encore, «Beyond the Object» de Barbara Rose (1975) figurent parmi les textes fondamentaux qui ont montré l'importance de la dématérialisation de l'objet d'art chez les artistes minimalistes et conceptuels des années 1960 et 1970. Si l'on affirme souvent que les œuvres chorégraphiques sont d'emblée immatérielles, elles le sont essentiellement par la durée éphémère de leur présentation, car sinon, il faut admettre la forte valorisation de la matière brute, soit le corps vivant. La littéralité et la matérialité des corps se trouvent exacerbées dans le travail des chorégraphes. Le commissaire Mathieu Copeland, qui réfléchit actuellement à cette contradiction, parle de «formes rematérialisées» dans le cadre des expositions chorégraphiques, par opposition aux «formes dématérialisées» associées à l'art conceptuel. ${ }^{13}$ Il remet en question l'exposition en tant qu'assemblage d'objets, ce qui l'amène à reconsidérer la matérialité des œuvres d'art et, par conséquent, des expositions, en fonction d'une «[...] compréhension, entre 
14. Ibid., P. 19

15. Ibid.

16. Voir Timothy McElreavy, «Paradise Lost/Paradox Found: Ma terializing a History of Conceptual Art», Blake Art Journal, vol. $61, n^{\circ} 4$, hiver 2002, p. 107-110.

17. Il existe une histoire intermittente de la danse au musée, mais les initiatives ont rarement perduré. Voir l'article de Claire Bishop «The perils and possibilities of dance in the museum». Dance Research Journal, vol. 46, $n^{\circ} 3,2014$, p. $62-76$.

18. Brian O'Doherty, Inside the White Cube. The Ideology of the Gallery Space, Santa Monica et San Francisco, The Lapi Press, 1986. Cette édition contient les trois premiers essais publiés initialement dans Artforum, vol. 14 et $15, \mathrm{~N}^{\circ} 3,7$ et 8, 1976. En 1981, un dernier article en continuité est publié: «The Gallery as a Gesture», Artforum, vol. 20, $\mathrm{n}^{\circ} 4$. Cet article fait partie de la nouvelle édition augmentée parue en 1999.

19. Tony Bennett, The Birth of the Museum. History, Theory, Politics, New York, Routledge, 1995 autres, du caractère éphémère et de la (non)-physicalité ou encore de l'(im) matérialité, qu'il s'agisse de l'absence et du vide, de la voix et du mot, du geste et du mouvement, et de leur chorégraphie intrinsèque». ${ }^{14}$ Plutôt que de considérer ces formes comme dématérialisées, il les considère comme des objets conceptuels desquels émergent des «possibilités de l'art, de la mémoire, et de la fabrique d'expositions». ${ }^{15}$ Ainsi, les chorégraphes développent des stratégies et des visées autres que celles des artistes conceptuels (Daniel Buren, Douglas Huebler, Joseph Kosuth, Sol LeWitt, Lawrence Weiner, On Kawara, par exemple) qui ont voulu éradiquer l'objet pour sortir des circuits institutionnels et marchands. Mais, de manière paradoxale, l'art conceptuel n'a pas aboli l'objet. Au contraire, il a introduit de nouveaux types d'artefacts sous forme de documents et d'archives, ${ }^{16}$ qui se retrouvent à leur tour dans des institutions artistiques et des marchés sous-jacents. De même, si les artistes visuels cherchent à échapper au musée, la trajectoire des chorégraphes et des danseurs est tout autre, car la danse, historiquement, est présentée à l'extérieur du musée, dans les théâtres. ${ }^{17}$ Pour les chorégraphes, c'est en se retrouvant au musée qu'il devient possible d'interroger le modèle muséal, en ce qu'il accorde peu de place aux corps vivants et encore moins à la danse. C'est dans cette perspective qu'il est possible d'envisager les pratiques chorégraphiques comme problématisant des questions d'ordre disciplinaire, institutionnel et social. Elles ébranlent la structure institutionnelle de l'intérieur tout en renouvelant la relation aux artefacts, aux documents et aux archives. Une telle approche participe au brouillage entre le travail artistique et commissarial initié par l'art conceptuel, et ouvre sur un engagement de l'artiste en phase avec les réalités du monde de l'art, où résister à l'institution signifie redéfinir les systèmes symboliques de l'art, mais aussi les systèmes de production et de diffusion.

\section{Deshistoires d'oset de chair}

La naissance du musée d'art s'inscrit en continuité avec la tradition des beauxarts et entérine un partage disciplinaire qui valorise des objets stables et permanents-artefact, peinture et sculpture. Les expériences proposées par les chorégraphes étant événementielles, éphémères et instables, elles s'opposent aux valeurs attribuées à l'objet artistique et contredisent son hégémonie. Le modèle du musée moderne tend, quant à lui, à privilégier une relation distancée avec les objets, et à dissimuler les procédures de fabrication. Comme le soutient Brian O'Doherty, dans son livre Inside the White Cube. The Ideology of the Gallery Space (1986 [1976]), ${ }^{18}$ le cube blanc de la salle d'exposition a contribué à décontextualiser les œuvres de leur lieu d'origine (lorsqu'elles sont, par exemple, retirées d'un bâtiment auquel elles étaient destinées), tout autant que des contingences matérielles ayant entouré leur réalisation. Tony Bennett souligne également, dans son livre The Birth of Museum (1995), ${ }^{19}$ que le musée moderne maintient une division entre les producteurs et les consommateurs de connaissances. En danse, il est difficile de dissocier le «faire» des arts vivants: c'est-à-dire que la pratique de l'interprète est l'œuvre elle-même qui se déroule sous les yeux du public. Dans cette perspective, l'insertion de la danse au musée permettrait de mettre de l'avant une pratique artistique qui se situe en amont de l'œuvre et dont l'œuvre matérielle est le produit. Plus 

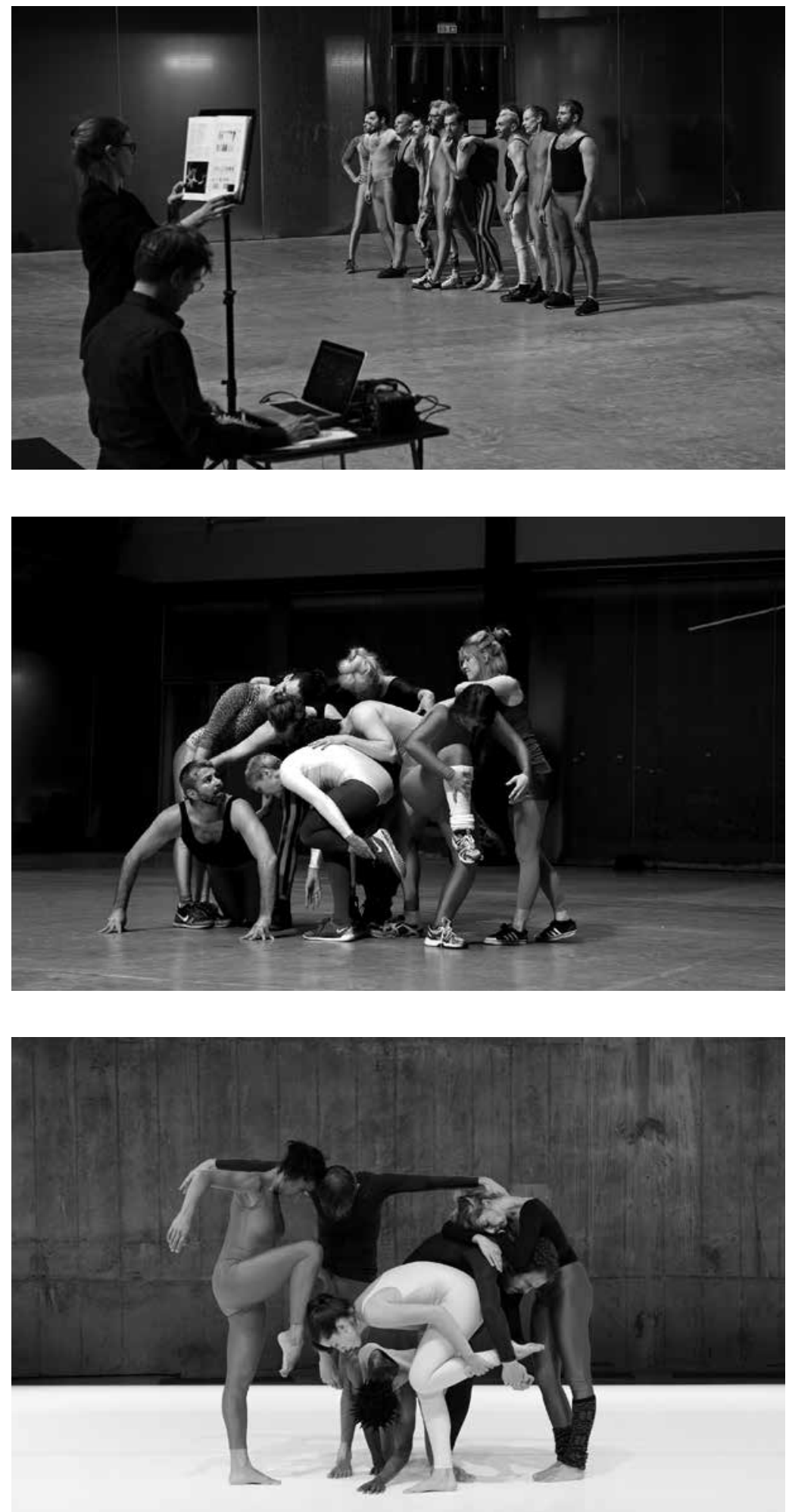

Figure 4. Boris Charmatz et le Musée de la danse, Roman Photo tiré de Flip Book, 2015, adapté par Olivia Grandville pour un groupe de non danseurs londoniens, Londres, Tate Modern. Photo: Brotherton Lock, (c) Tate.

Figure 5. Boris Charmatz et le Musée de la danse, Roman Photo tiré de Flip Book, 2015, adapté par Olivia Grandville pour un groupe de non danseurs londoniens, Londres, Tate Modern. Photo: Brotherton Lock, (c) Tate.

Figure 6. Boris Charmatz et le Musée de la danse, Flip Book, 2012, en performance lors de l'événement The Tanks: Art in Action, Londres, Tate Modern. Photo: Tate, (c) Tate. 
20. Jean-Pierre Cometti, Art et facteurs d'art: ontologies friables, Rennes, Presses universitaires de Rennes, 2012, p. 33.

21. Marcella Lista, «Play Dead, Museums, and the Time-Based Arts", Dance Research, vol. $46, \mathrm{n}^{\circ} 3$, 2014, p. 23.

22. Ibid. encore, la danse attire l'attention sur la manière dont se développe un champ de pratique plus empirique: les modalités de transmission orales et corporelles, l'implication humaine et ses spécificités ou encore le travail en communauté. On peut avancer, à la suite du philosophe Jean-Pierre Cometti, que cette importance de la pratique artistique vivante s'inscrit dans un paradigme qui privilégie l'action et l'événement, ce qui conduit à faire de la performance «le modèle et l'instrument d'une reconception de l'art». ${ }^{20}$ L'objet n'est plus la finalité de l'exercice d'un savoir-faire. Toutefois, il faut considérer aujourd'hui le travail fait sur la matérialité, qu'il s'agisse de produire un document photographique comme une œuvre indépendante issue elle-même d'une pièce dansée ou performée, ou encore, de montrer les différents processus menant à une forme matérielle ou de faire intervenir des médiums en relation avec les corps. Loin d'une vision dualiste, la danse au musée initie un dialogue entre le corps vivant et les formes matérielles.

Cette temporalité événementielle, c'est-à-dire conçue pour des moments déterminés en relation avec le public, va au-delà des fonctions habituelles du musée centrées sur une collection matérielle et sur l'exposition de l'œuvre. C'est pourquoi la danse permettrait de mettre à jour des récits plus près de l'événementiel et, par conséquent, plus éloignés des approches téléologiques en histoire de l'art. L'historienne de l'art Marcella Lista affirme que les arts vivants au musée marient deux normes d'économies temporelles, qu'elle définit comme suit: «[...] on the one hand the linear course with its one-way narrative spectacularization; on the other the time of events, designed at set times to involve the audience in something beyond the formats of collection and display». ${ }^{21}$ Ainsi, les corps vivants bouleversent à différents niveaux les processus de conservation par lesquels les musées contribuent à l'histoire de l'art: «In this sense, dance can challenge the spectacularization of museums: it endeavours to affect the forms of that very historical narrative that shape the institution and the visitor's experience».22 Dans cette perspective, le travail en arts vivants permet de repenser la temporalité linéaire du récit historique valorisé par le musée.

Par les manières de faire interagir les pratiques et les médiums, le travail de Boris Charmatz génère des formes de narrativité et des récits complexes qui en appellent souvent aux histoires des pratiques. Une pièce comme Flip Book montre un réinvestissement humain et démocratique du matériel archivistique. Elle décline une trame chorégraphique à partir des photographies du livre Merce Cunningham, un demi-siècle de danse (1997) de David Vaughan, l'archiviste de la Merce Cunningham Company. Flip Book, (fig. 4) comme son nom l'indique, s'inspire des petits livres illustrés dont les gestes sont décomposés sur chacune des pages, de telle sorte que lorsqu'il est feuilleté rapidement, le livre donne l'illusion d'un dessin animé. La performance se décline selon trois protocoles réalisés avec des groupes distincts, où sont reprises une à une les photos du livre: Roman Photo, avec des amateurs ou non-danseurs; | fig. 5| Flip Book, avec des danseurs professionnels: | fig. 6| 50 ans de danse, avec les anciens interprètes de la Merce Cunningham Dance Company.

Dans le segment Roman Photo, le chorégraphe enseigne à des non-danseurs la partition chorégraphique devant le public, en récréant physiquement les photographies avec le groupe. Le projet relève de l'actualisation du matériel 
23. Nous traduisons ici l'expression originale anglaise, "operational field of projected total knowledge». Richard Thomas, cité par Rebecca Schneider dans Performing Remains: Art and War in Times of Theatrical Reenactment, Abington Oxon, Routledge, 2011, p. 100. 24. Ibid. archivistique par le biais de la danse et de l'expérimentation pour tous, ce qui inverse le processus muséal traditionnel, de l'œuvre vers l'archive et sa conservation. La performance a lieu dans l'atrium du MoMA, le livre 50 ans de danse est bien à la vue du public sur un lutrin ou entre les mains de Charmatz. Ici, l'archive «fait œuvre» par le biais de son incarnation corporelle. Dans l'ensemble, Flip Book exacerbe ce va-et-vient entre l'archive et l'œuvre, les deux étant constamment rejouées d'un segment de la pièce à l'autre: chaque groupe doit rejouer la partition constituée des photographies selon des procédures qui diffèrent à chaque fois. À ce titre, le segment 50 ans de danse est particulièrement pertinent. L'ancienne danseuse de la Merce Cunningham, Valda Setterfeld, est invitée à discuter de son expérience avec le chorégraphe, mais aussi de l'histoire de la compagnie, de sa trajectoire personnelle ou encore de son expérience avec Charmatz. Ici, le récit historique se mêle à l'histoire personnelle de la danseuse, et intègre également les conditions de vie et de travail au sein de la compagnie, tout comme les procédés de création de Cunningham. Ce discours resitue le travail du chorégraphe dans l'histoire des arts, mais aussi en appelle aux spécificités de ce travail et à son ancrage social: il met particulièrement à l'avant-plan la vie au sein d'une compagnie permanente, ainsi que le dévouement et le travail acharné des danseuses et danseurs. Tout au long de l'échange, on assiste simultanément à un atelier pratique, d'un segment de Flip Book: sur le plancher de danse, disposés au sol, les danseurs commencent à s'échauffer un à un. À l'arrière sur le mur, sont projetées des archives visuelles, présentant par exemple la captation vidéo d'une présentation antérieure de 50 ans de danse que Setterfeld commente en direct. Ainsi, la mémoire vivante (Setterfeld) et la documentation matérielle (la vidéo) entrent en dialogue. Les commentaires live de Setterfeld donnent à comprendre l'incapacité de certains documents à rendre compte de la complexité de l'expérience vécue dont seule l'interprète peut témoigner. Soulignons que le public n'intervient pas en bout de course quand l'œuvre est achevée, mais participe selon différentes modalités à la trajectoire de l'œuvre, qu'il s'agisse de participer à l'enseignement de Charmatz ou d'adresser des questions à Setterfeld pendant qu'elle relate son histoire.

Ce projet transforme les méthodologies muséales et brouille l'ordre du musée qui sépare la production de la présentation, la présentation de la réception, et ainsi de suite. L'archive y est repensée en tenant compte de l'expérience des danseurs et des spectateurs. Les procédures de fabrication montrent les limites de l'archive tout en participant à l'œuvre. Il s'agit de revaloriser une tradition orale qui est diminuée dans une culture où l'archive visuelle prime par sa stabilité et sa persistance dans le temps. Dans son ouvrage Performing Remains: Art and War in Times of Theatrical Reenactment (2011), Rebecca Schneider remarque comment les archives constituent «des champs opérationnels logiques de savoir total», ${ }^{23}$ dont la loi dérive de son autorité pour commander. Schneider utilise notamment la métaphore de la chair et des os, comparant les os à l'archive: ce qu'il reste quand la chair s'est décomposée (la relique). ${ }^{24}$ La chair, quant à elle, est renvoyée à la matière instable et dépréciée, car périssable. L'approche de Charmatz vise à remettre de la chair autour de l'os et à défendre la singularité du savoir performé. Schneider affirme que plusieurs pratiques performées et 
25. Rebecca Schneider, «Performance remains again», dans Gabriella Giannachi, Nick Kaye et Michael Shanks, dir., Archaeologies of Presence: Art, Performance and the Persistence of Being, Londres, Routledge, 2012 , p. 67.

26. Ibid., p. 76 .

27. Marcella Lista, «Play Dead: Dance Museums, and Time-Based Arts», Dance Research Journal, vol. 46, $\mathrm{n}^{\circ} 3,2014$, p. 21 codifiées-primitives, populaires, folkloriques, reconstitutions historiques, danse et théâtre-gardent justement la mémoire vivante et que leur transmission par le corps-à-corps assure leur pérennité, notamment par l'affect qui les ancre plus solidement dans notre mémoire. En ce sens, elle explique comment plusieurs approches ont préconisé que le corps ne puisse préserver une mémoire: "Such statements assume that memory cannot be housed in a body and remain, and thus that oral storytelling, live recitations, repeated gesture, and ritual enactments are not practices of telling or writing history ${ }^{25}$ Or, des philosophes de l'histoire, dont Pierre Nora, vont inclure la mémoire et les pratiques collectives dans une «nouvelle histoire». Schneider envisage à ce titre la performance comme un médium dans lequel «disappearance negotiates, perhaps becomes, materiality» ${ }^{26}$ Elle fait valoir que les savoirs, qu'ils soient matériels ou performés, nécessitent une architecture, un dispositif, qui engage à son tour des actions. Cette approche nous invite à considérer les processus et les actions comme intimement liés au savoir. La danse se transmet de manière orale et d'un corps à l'autre. Lorsque Charmatz enseigne la partition chorégraphique aux non-danseurs, il «expose» ce processus de transmission orale en même temps qu'il informe les participants des spécificités stylistiques propres au travail de Cunningham, dont la compréhension passe par l'expérimentation. Charmatz raconte à sa manière le travail de Cunningham. Il le commente librement en mettant en relation plusieurs interprétations possibles. La valeur de l'œuvre se fonde sur l'expérience partagée, plutôt que sur un savoir-faire menant à un objet qui persiste dans le temps. Les processus mêmes de production de Flip Book se fondent sur la passation de la pièce, d'un segment à l'autre, d'un livre photographique comme partition à des corps dans l'espace, ce qui crée une co-dépendance des corps vis-à-vis des archives photographiques. Dans l'espace muséal, la portée critique de cette chorégraphie est indéniable au regard des méthodes de conservation et de commissariat qui, elles-mêmes, sont historiquement définies autour de valeurs liées à l'œuvre d'art comme objet.

Dans son ensemble, Flip Book donne à comprendre l'héritage artistique et humain de Cunningham à partir d'une histoire dont les filons narratifs se multiplient: par le biais des témoignages, de l'enseignement, des commentaires et des expérimentations. Bref, le dispositif nous permet de comprendre la complexité de la mémoire qui se cristallise dans le cadre de la danse par un travail en communauté, dont Charmatz exemplifie les modes de transmission et de circulation. Comme le souligne l'historienne de l'art Marcella Lista, la danse fait entrer dans les musées une mémoire plus libre et émancipée des codes de conservation établis: «designed at set times to involve the audience in something beyond the formats of collection and display». Elle poursuit:

By introducing labyrinths, cross-rhythms, and breaks in time, dance engages in a fertile experimentation with art history through its transformation into a freer museal memory of the established codes of conservation. It also calls upon performers, spectators, and the institutional powers-that-be to open up some new uses of shared time that have not so far been thought of. ${ }^{27}$

Le format événementiel de l'exposition multiplie les trames narratives et les lectures possibles. Il fait éclater toute lecture univoque de l'œuvre d'art. Si l'événement est, dans une certaine mesure, défini par les contraintes 
28. Paul Ricœur, «Le retour de l'événement", Mélanges del'École française de Rome, t. 104, $\mathrm{n}^{\circ}{ }^{1}$, 1992, p. 35 .

29. Michel Foucault, «Nietzsche, la généalogie, l’histoire», dans Hommageà Jean Hyppolite, Paris, Presses universitaires de France, 1971, p. 145-172.

30. Ibid., p. 155

31. Ibid., p. 170. (protocoles, règles, situations) que désigne l'artiste, il demeure que les formes événementielles (atelier, conférence, discussion) privilégient l'improbable et la singularité de chacune de leur occurrence. L'événement artistique interroge nos approches de l'œuvre d'art et nos histoires des arts, comme le souligne Lista, en renouvelant constamment nos manières de les inscrire dans nos mémoires. Flip Book met aussi particulièrement en jeu la mémoire physique du spectateur. L'événement résiste à une prise en charge traditionnelle, puisqu'il passe en grande partie par des interprètes actifs, ceux qui forment l'œuvre, mais aussi ceux qui en font l'expérience: les spectateurs. Ce qui correspond pour Paul Ricœur à «l'histoire effective», celle qui appartient à tous, puisque nous la faisons et la subissons tous individuellement en tant qu'humains. L' «histoire effective» permet à la conscience individuelle de conjuguer l'espace d'expérience (le passé incorporé à l'expérience du présent) et l'horizon d'attente (le souci du futur donnant une directionnalité au présent lui-même): «Voilà pourquoi l'événement qui surgit réunit les deux significations majeures du mot histoire: série des événements en train de se produire, et récit des événements par ceux qui, ne l'ayant pas vécue, la reconstruisent».. ${ }^{28}$ On peut avancer que Flip Book réunit aussi ces deux significations de l'histoire. La pièce relève le défi de construire un récit qui intègre l'interprétation en convoquant un équilibre entre le réel et sa représentation. Cette position s'oppose aux conceptions de l'histoire tournées vers une origine idéalisée. Elle se rapproche de la généalogie, telle que formulée par Michel Foucault à la suite de Friedrich Nietzsche, qui considère l'histoire comme fondamentalement plurielle. Dans «Nietzsche, la généalogie, l'histoire» (1971), ${ }^{29}$ Michel Foucault revendique la discontinuité de l'événement comme fondement de la généalogie. En effet, il avance que l'événement se caractérise par une singularité irréductible et hors processus. Il aborde l'événement comme ce qui fait resurgir la pluralité des histoires, ce qui fait signe vers l'hétérogénéité des mondes, des espaces, des perspectives. À partir de l'événement, il repense l'histoire, mais en substituant à la catégorie jusqu'alors incontestée de la continuité celle de la «dissociation systématique». ${ }^{30}$ L'événement y est perçu non pas comme ce qui se dissout dans une construction linéaire, mais bien comme une «myriade d'événements enchevêtrés» et "perdus». ${ }^{31}$ L'événementy est un motif de discontinuité. La généalogie s'oppose à une conception de l'histoire téléologique chez Foucault, ce qui mène à reconsidérer l'histoire comme fondamentalement plurielle. Dans cette perspective, Flip Book confronte l'événement à ce qui reste: l'archive, le document ou la trace matérielle. Il fait de ces éléments des outils ou des instruments pour imaginer de nouvelles modalités de faire, d'être et de dire avec eux et autour d'eux des histoires.

\section{Entre objet et sujet: la singularité du danseur}

Le travail de Xavier Le Roy tout comme celui de Boris Charmatz se construit autour d'un certain nombre de procédures qui offrent la possibilité de révéler le danseur dans sa position de sujet. Cela signifie que la chorégraphie n'est pas orientée vers des compétences qui contribuent à l'élaboration formelle du matériel chorégraphique, mais plutôt vers l'exercice de différents modes d'action des danseurs sur eux-mêmes et sur leur espace commun de création. Le corps du danseur se trouve lui aussi interrogé comme un site idéologique 

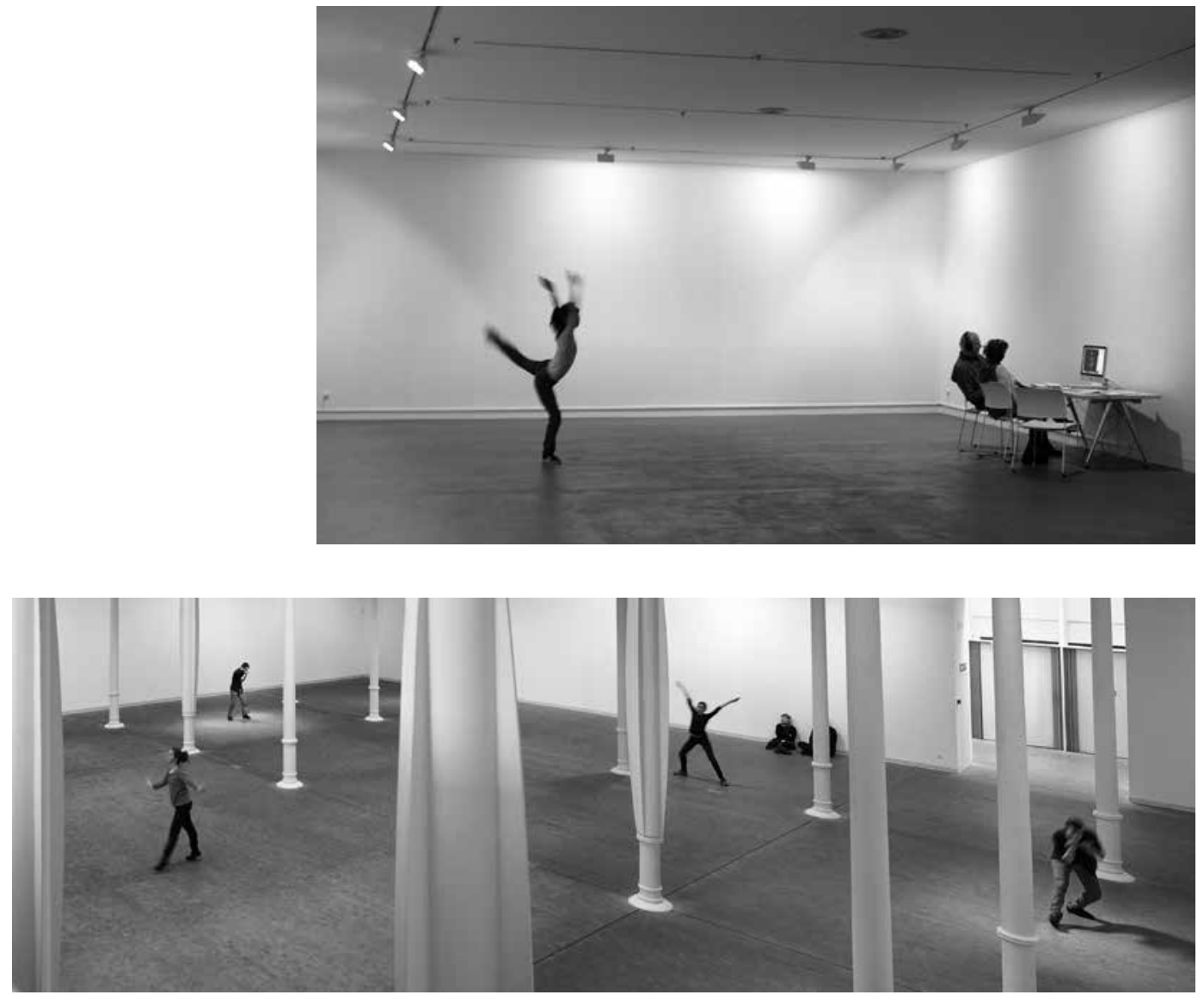

Figures 7-9. Xavier Le Roy, "Rétrospective», 2012, Barcelone, Fundació Antoni Tàpies. Photo:

Lluis Bover, () Fundació Antoni Tàpies.

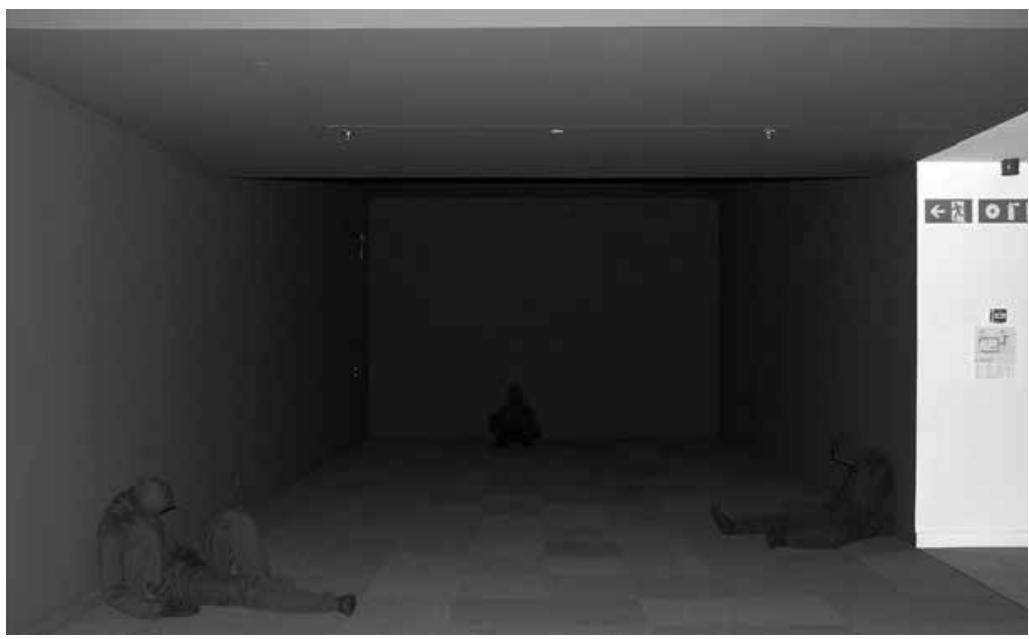


32. Les pièces antérieures étaient: SelfUnfinished (1998), Produit de Circonstances (1999), Le Sacre du Printemps (2007), Narcisse Flip (19941997), Self Interview (2000), Giszelle (2001), Sans Titre (2005) et Produit d'autres circonstances (2009).

33. Produit de circonstances est une conférence autobiographique de Xavier Le Roy afin d'expliquer son passage professionnel du domaine des sciences au domaine de la danse. S'y mêlent plusieurs circonstances de sa vie personnelle.

34. Sans-Titre (2012) implique deux figures masquées aux physiques similaires qui se déplacent ensemble dans l'obscurité. La situation remet en question la reconnaissance de ce qu'ils font et de ce qu'ils sont. La pièce utilise la conjonction de l'obscurité, des mannequins, des marionnettes et des représentations humaines pour rechercher des zones d'indécision.

35. Bojana Cvejić, «Retrospective de Xavier Le Roy, chorégraphier un problème et un mode de production», dans Cvejić, dir., op. cit., p. 10. qui correspond à sa formation technique liée à ses fonctions artistiques au sein des œuvres. En opposition à une fétichisation du corps, on donne à comprendre les processus de subjectivation dans les domaines matériel et discursif. «Rétrospective» (2012) est exemplaire à ce titre, puisque la pièce fait appel librement à la mémoire des danseurs: ceux-ci doivent réinterpréter les solos de Le Roy en échos avec leurs histoires personnelles. Le chorégraphe revisite ainsi le format de la rétrospective, en faisant de la reprise de ses solos antérieurs ${ }^{32}$ le prétexte pour mettre en évidence le travail et la subjectivité des interprètes.

Dans l'atrium nu, | fig. 7 | se trouvent quatre danseuses ou danseurs qui, chacune ou chacun, présente un extrait d'une des chorégraphies de Le Roy, selon trois modalités différentes: en incarnant, immobile, une image tirée d'une chorégraphie antérieure; en produisant en boucle une séquence; en interpellant le spectateur et en relatant sans interruption sa propre histoire professionnelle et personnelle à la manière de la pièce Produit de circonstances..$^{33}$ Les quatre artistes livrent leur propre interprétation des chorégraphies, c'està-dire que leur subjectivité participe pleinement à la proposition. Les actions se déroulent en simultanée. Les spectateurs sont libres de circuler, mais dès qu'ils font leur entrée dans l'espace, les artistes sortent et reviennent, comme si le jeu reprenait du début, ce qui intensifie l'idée de boucle propre à la partition originale. À la salle principale s'ajoutent deux autres salles dans lesquelles l'exposition se poursuit. L'une, que Le Roy nomme «la fabrique» ou le "studio», montre le travail en cours des danseurs sur leurs rétrospectives individuelles: les spectateurs peuvent y consulter des archives vidéo (présentant le corpus), | fig. 8 | de même que discuter avec un danseur. L'autre salle est plongée dans l'obscurité et présente des mannequins grandeur nature, reliques de la pièce Sans titre (2005). ${ }^{34}$ | fig. 9 |

La commissaire Bojana Cvejic définit bien les enjeux qui président à l'exposition en relation avec l'espace muséal:

\footnotetext{
La création de «Rétrospective» commence par une réflexion critique sur les conditions qui structurent le champ chorégraphique étendu au musée: le statut de l'œuvre en tant qu'objet, événement ou dans la définition qu'en donne Xavier Le Roy en tant qu'une «situation mise en scène au moyen d'artifices» à laquelle participe des choses, concepts, images, rencontres, histoire et durées; une temporalité intensifiée grâce à l'appareil de représentation du musée, lequel diffère de celui du théâtre; le danger de déposséder la performance des conditions matérielles de sa production. ${ }^{35}$
}

Cette situation exacerbe la tension entre les modes de représentation du musée, qui tendent à vouloir figer les formes chorégraphiques en mouvement, et les modes de représentation du théâtre, qui exigent une action continue. C'est ce que met en scène ici Xavier Le Roy. Alors que le musée a tendance à vouloir inscrire les œuvres dans un récit historique, dans un contexte et un temps défini, la forme temporelle privilégiée par Le Roy suppose une constante réappropriation de l'œuvre à travers la partition et la mémoire des interprètes. Ainsi, plutôt que de faire de la rétrospective un lieu de commémoration qui fige l'œuvre d'un artiste dans le temps, Le Roy met en évidence la réappropriation constante de l'œuvre pour qu'elle perdure dans le temps. Cette approche de la danse diffère de la tendance actuelle et dominante dans les musées qui vise à recréer des œuvres historiques. Comme le souligne Claire Bishop, dans son article «The Perils and Possibilities of Dance in the 
Figure 10. Xavier Le Roy, "Rétrospective», 2014, Paris, Centre Pompidou. Photo: Hervé Véronèse, (c) Centre Pompidou.
36. Bishop, op.cit., p. 107.

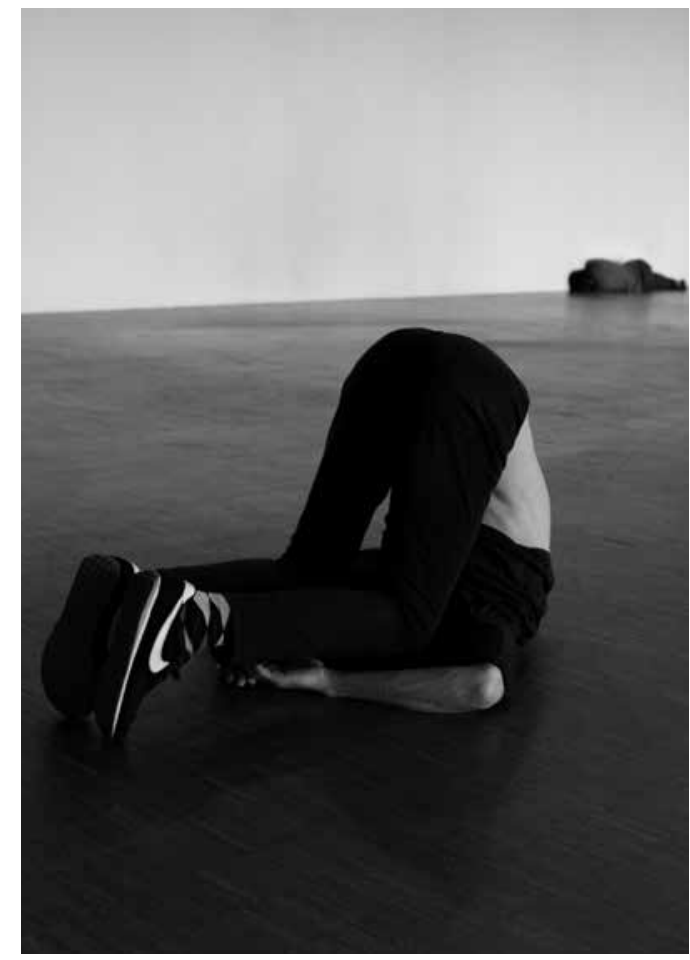

Museum ", la recréation d'œuvres en danse est une posture conservatrice, qui n'embrasse pas pleinement le potentiel et l'actualité des arts vivants.

De manière analogue à Charmatz, Le Roy impose une approche archivistique pensée en résonance avec le travail de danse, c'est-à-dire que la fonction des interprètes est mise à l'avant-plan: on passe d'une archive inerte à une archive vivante. Dans le «studio», les archives vidéo servent à montrer le travail du danseur, ou encore, à susciter l'interprétation active et critique des spectateurs de l'exposition. Les conversations possibles entre danseurs et spectateurs qui accompagnent la présentation des vidéos font primer l'expérience humaine. Dans la seconde salle, les reliques-mannequins (qui représentent des humains) provenant de Sans titre ne sont pas éclairées, elles sont dans l'obscurité. Dans cette perspective, cette salle apparaît comme un commentaire critique sur l'archive dans la mesure où, sans interprétation ou réactivation, l'archive devient obscure. Comme le souligne Claire Bishop, «Rétrospective» montre «[...] le désir de préserver la vie des œuvres existantes et de les ouvrir pour les transformer (plutôt que de les archiver dans le passé) en se servant de l'exposition même comme d'un médium destiné à présenter le travail». ${ }^{36}$ Véritable machine chorégraphique en constante activité, «Rétrospective» part du double constat de l'impossibilité de recréer les chorégraphies et de la possibilité de se les remémorer.

Ainsi, le statut de ceux qui «incarnent» les pièces est aussi interrogé, puisqu'il s'agit de sujets qui tendent à être objectivés en même temps qu'ils résistent à cette objectification. Dans la salle principale, la répétition d'une séquence en boucle ou encore l'immobilité du corps | fig. 10| rapproche les 
danseurs d'une forme sculpturale ou objectale. Par ailleurs, la discussion et la prise de parole sur leur vie individuelle en font des sujets: chaque danseur est amené à relater aux visiteurs (sous forme de conversation) son parcours professionnel et personnel en relation avec le travail de Le Roy. Ainsi, l'exposition met à nu des tensions, ou des ambiguïtés, entre les objets et les sujets. En ce sens, Le Roy interroge le statut des individus:

[...] les individus doivent-ils devenir des objets? Ou est-ce le visiteur qui les objective? [...]
Peut-être que cette dynamique est évidente pour le spectateur et le performeur dans la
mesure où elle est devenue invisible, car faisant habituellement partie du contrat impli-
cite qui régit les performances se déroulant dans un théâtre. En revanche, dans un espace
d'exposition, si un performeur interprète littéralement un objet, cela devient ambigu. En
regardant un corps comme il le ferait avec un objet, le visiteur pourra être amenéà s'inter-
roger sur la façon dont il considère d'autres choses inanimées, comme objet. Cela m'inté-
resse également de questionner dans quelle mesure devenir un objet n'est pas simple-
ment «mal»-en fonction de la décision que vous prenez-, mais comment cela peut offrir
un autre type de subjectivation, plutôt qu'un assujettissement ou subjugation à une loi. ${ }^{37}$

Les relations entre les corps et l'espace d'exposition mettent en évidence la manière dont les limites entre le sujet-objet se définissent et s'élaborent dans ce contexte. La proximité des spectateurs, leur mobilité et l'abolition de la scène créent des zones d'ambiguïté: elles déstabilisent les cadres perceptuels-du musée, de la scène et de la vie ordinaire-ce qui invite le spectateur à s'interroger. Les habitudes perceptives liées aux espaces de présentation peuvent mener à des interprétations radicalement différentes de notre rencontre avec l'œuvre et à une lecture critique du dispositif de présentation. D'une part, la prise de parole des danseurs et les discussions qu'ils peuvent engager librement avec les spectateurs les font entrer dans un registre plus

37. Xavier Le Roy, «La première conversation à propos de Rétrospective», entretien par l'auteure Bojana Cvejic, dans Cvejić, dir., op. cit., p. 268.

38. Plus largement, on peut avancer que la conférence-performance est devenue un genre en soi, d'abord exploité dans l'art conceptuel et l'art de la performance par le biais d'expérimentations liées au langage. Depuis les années 1990, on la voit également apparaître dans le domaine de la danse contemporaine. Aujourd'hui, comme le suggère Rike Frank, ce terme désignerait un ensemble de pratiques assez hétérogènes qui sont préoccupées par «la valeur croissante de la conversation comme moyen de production de connaissances dans un hypercommunicatif et le rôle des artistes-chercheurs contemporains au sein de l'éducation». Voir Rike Frank, «When Form Starts Talking: On Lecture-Performances», Afterall, $n^{\circ} 33,2013$, p. 4-14.

39. Les Laboratoires d'Aubervilliers: des formes de vie, www. desformesdevie.org/fr/page/ formes-vie-par-jean-pierre-cometti (consulté le 1er octobre 2017). social, alors que, d'autre part, leur immobilité et la reprise en boucle d'une séquence tendent à les objectiver. Ainsi, l'exposition joue constamment sur les recadrages de l'œuvre, créant une forme d'indécidabilité entre la représentation et le réel, l'objectification et la présentation du danseur comme sujet.

La conférence-performance, qui est un format exploité à la fois par Le Roy et Charmatz, devient à son tour une stratégie commissariale pour mettre en évidence l'engagement du chorégraphe et/ou des interprètes dans leur travail artistique. ${ }^{38}$ Cette stratégie permet de révéler les «formes de vie», selon le terme de Jean-Pierre Cometti, sous-jacentes au travail des artistes. Le discours des danseurs met en jeu simultanément l'histoire des arts, leur histoire personnelle, leur relation à l'art, les spécificités de leur pratique artistique et leur ancrage social. Cette posture a un fort potentiel critique face à l'objectivité de l'œuvre d'art et à sa séparation des processus de création réels qui s'inscrivent dans le cours de la vie ordinaire. C'est ainsi que Cometti propose d'envisager l'œuvre d'art en continuité avec les modes de production artistique:

[...] penser en termes de formes de vie ou s'interroger sur le rapport qui conjugue nos pratiques ou nos formes de pensée à une (ou des) forme(s) de vie, c'est prendre la mesure de tout ce qui est impliqué, incorporé, présupposé dans ce qu'elles présentent en apparence de plus autonome, autrement dit renouer le lien qu'elles n'ont probablement jamais complètement dissous avec la culture et les pratiques humaines communes sous toutes leurs formes. C'est aussi ou ce peut être, du même coup, en proposer d'autres descriptions, d'autres expériences, plus riches ou plus fécondes, en pensant par exemple, jusqu'à les y inclure, aux processus qui entrent dans la production artistique ou, sur un autre plan, aux conditions d'activation des objets esthétiques. ${ }^{39}$ 
40. Jacques Rancière, Lepartage du sensible, Paris, La Fabrique, 2012, p. 24.

41. Ibid., p. 72-73.

42. André Lepecki, Singularities, Dance in the Age of Performance, New York, Routledge, 2016, p. 18.

43. Dorothea von Hantelmann, «When You Mix Something, It's Good to Know Your Ingredients: Modes of Addressing and Economies of Attention in the Visual and Performing Arts», dans How to Frame. On the Threshold of Performing and Visual Arts, Berlin, Sternberg Press, 2016, p. 52
Le processus artistique (pour les artistes comme pour les spectateurs) devient donc tout aussi important que l'œuvre. Il est ce qui permet l'émergence des trames narratives de l'œuvre, plus près d'une expérience pragmatique de l'art. Dans cette perspective, ces partis pris mènent à comprendre autrement le savoir issu de l'art, plus près d'un partage d'expérience ou du «sensible», que Jacques Rancière définit comme le «découpage sensible du commun de la communauté, des formes de sa visibilité et de son aménagement [...]». ${ }^{40}$ En cela, la mise à vue de la pratique artistique et le partage de celle-ci avec le spectateur, tout comme l'appropriation d'une histoire de l'œuvre et de l'art, témoignent «d'une recomposition du visible, du rapport entre le faire, l'être, le voir et le dire». ${ }^{41}$ Cette approche mène à considérer autrement l'inscription de la pratique artistique qui ne repose plus sur des connaissances techniques et sensibles. Il s'agit de montrer les dimensions cognitives et discursives qui l'accompagnent.

Cette dimension mène à requalifier les interventions chorégraphiques comme ayant une portée commissariale engagée, car les corps vivants, par leur singularité, résistent aux formes commissariales centrées sur l'objet. Elles résistent aussi au contrôle qu'exercent les institutions sur la conservation et l'exposition des objets pérennes. Le théoricien André Lepecki va plus loin en affirmant que la danse dans ses singularités s'oppose à la rationalité néolibérale à travers ses traits les plus fondamentaux: l'éphémère, la corporalité, la précarité, la partition, la performativité et la performance du travail affectif. La danse pourrait ainsi s'inscrire dans un contre-mouvement et un contre-discours:

Once we take all the aspects just outlined (which, again, are all constitutive of dance singularity as artistic practice), it becomes clear why dance has become an energizing and catalyzing element in contemporary art and in contemporary critical thought. Dance experiments with acute critique of current conditions of existence. In this sense, dance's most significant singularity is its capacity for gathering and articulating the set of defining problems in neoliberal production of subjectivity as it produces countermoves and counter discourses. Because the singularity that transforms spaces of circulation into spaces of freedom and of moving political potentialities has a specific name: the dancer. ${ }^{\mathbf{4 2}}$

La danse contemporaine telle que définie ici tient à la mise en action de l'œuvre par le danseur qui exerce une forme de liberté. Le travail de Charmatz et de Le Roy met en lumière ce «contre-discours» par la mise à l'épreuve du musée par les corps. L'historienne de l'art Dorothea von Hantelmann affirme que les expositions en arts vivants participent à une dimension relationnelle qui se situe a contrario des valeurs véhiculées par l'espace de la galerie et des idées de la société moderne: l'individualisme, le productivisme, la notion évolutive du temps et du progrès, le désengagement des individus et des objets dans leurs interactions. ${ }^{43}$ Elle lie l'entrée des arts vivants dans l'espace de la galerie aux changements sociaux actuels, qui donnent une prévalence à l'activité relationnelle et aux processus sociaux sur la production d'objets. Il apparaît alors nécessaire de relier ensemble ce qui a été séparé, le vivant et le non-vivant, pour faire face aux conséquences écologiques, sociales et économiques du monde moderne. Von Hantelmann déclare aussi que l'intérêt pour les arts vivants correspond à leur capacité de rassemblement dans un contexte où il y a démocratisation de la culture. Selon elle, l'une des différences fondamentales dans les modes d'adresse des institutions en arts visuels et en arts de 
la scène est liée au public: l'expérience visuelle est destinée à l'individu dans le cadre d'une galerie, tandis que le théâtre s'adresse à une collectivité.

Dans cette perspective, Charmatz et Le Roy mettent en évidence une dialectique entre l'œuvre-objet et l'œuvre-sujet en allant au-delà d'une simple dichotomie pour rassembler ce qui a été séparé. Leurs expositions chorégraphiques redéfinissent ainsi les pôles de la production et de la réception au sein de leur travail artistique en faisant de la médiation une des composantes de leur œuvre. Aujourd'hui, on autorise de plus en plus les artistes à utiliser le musée comme support et matériau de l'œuvre, leur reconnaissant une certaine compétence sur les questions relatives à l'exposition. Chez Charmatz et Le Roy, cet engagement commissarial se formule à travers la mise en place d'un contre-discours (André Lepecki) et d'une contre-histoire (Rebecca Schneider), c'est-à-dire que les chorégraphes proposent de nouvelles lectures de l'histoire à partir de la mémoire (rattachée à l'oralité et à la transmission au sein d'une communauté). Ainsi, ils contrecarrent la séparation entre le corps et les objets préconisée par le musée afin de rétablir un dialogue entre la culture matérielle et corporelle. Par le truchement de ce dispositif, le travail des danseurs et leurs relations avec les formes de vie sociale et politique deviennent explicites. Le musée, comme système de représentation de l'art, a traditionnellement valorisé l'objet esthétique et favorisé un rapport détaché de la culture plutôt qu'un rapport relationnel, comme on l'a vu ici avec la danse. L'engagement de Charmatz et de Le Roy se traduit par une volonté de réformer ce type de dispositif muséal, en redonnant préséance à la pratique vivante et à la recherche artistique. Car le «corps incarné» de la danse vient reformuler un espace de liberté. Cette démarche commissariale culmine par la prise en charge complète du musée, comme c'est le cas de Charmatz avec le Musée de la danse. Dans ce contexte, la participation du chorégraphe au musée lui-même permet de définir ses propres règles de fonctionnement, qu'elles soient esthétiques, symboliques ou économiques. Cet espace de liberté n'est pas un lieu complètement émancipé des systèmes économiques et politiques, mais il permet de trouver des moyens d'interagir et de concilier ces systèmes plus librement. Il s'agit d'exposer de manière explicite leur emprise et de renforcer les valeurs symboliques et sociales liées à l'art en cherchant des moyens pour les mettre au service des communautés artistiques, plutôt que de subordonner la dimension artistique à celle politique et économique. Le commissariat en arts vivants se situe ainsi au carrefour d'enjeux liés à l'économie néolibérale et au spectaculaire, et d'un commissariat engagé par le biais de démarches artistiques critiques qui redéfinissent notre relation à l'art et à l'institution. Dans cette perspective, les expositions chorégraphiques et performatives mènent à un renouvellement des modalités institutionnelles, tout en nous sensibilisant aux questions éthiques qui touchent aux enjeux du travail immatériel. q 